\title{
BMJ Open Initiation and persistence with dual antiplatelet therapy after acute myocardial infarction: a Danish nationwide population-based cohort study
}

\author{
Anders Green, ${ }^{1,2}$ Anton Pottegård, ${ }^{3}$ Anne Broe, ${ }^{3}$ Thomas Goldin Diness, ${ }^{4}$ \\ Martha Emneus, ${ }^{2}$ Pål Hasvold, ${ }^{4}$ Gunnar H Gislason ${ }^{5}$
}

To cite: Green $A$,

Pottegård A, Broe A, et al. Initiation and persistence with dual antiplatelet therapy after acute myocardial infarction: a Danish nationwide population-based cohort study. BMJ Open 2016;6: e010880. doi:10.1136/ bmjopen-2015-010880

- Prepublication history and additional material is available. To view please visit the journal (http://dx.doi.org/ 10.1136/bmjopen-2015010880).

Received 28 December 2015 Revised 9 April 2016 Accepted 20 April 2016

CrossMark

For numbered affiliations see end of article.

Correspondence to Professor Anders Green; anders.green@dadlnet.dk

\section{ABSTRACT}

Objectives: The study investigated dual antiplatelet therapy (DAPT) patterns over time and patient characteristics associated with the various treatments in a myocardial infarction (MI) population.

Design: A registry-based observational cohort study was performed using antecedent data.

Setting: This study linked morbidity, mortality and medication data from Danish national registries.

Participants: All 28449 patients admitted to a Danish hospital with a first-time $\mathrm{Ml}$ and alive at discharge from 2009 through 2012 were included.

Primary and secondary outcome measures: Primary outcome was initiation of DAPT and secondary outcomes comprised persistence in DAPT treatment and switches between DAPT treatments.

Results: The overall proportion of patients prescribed DAPT increased from 68\% (CL 95\% 67-69\%) to $73 \%$ (CL 95\% 72-74\%) from 2009 to 2012. For treatment of patients with and without percutaneous coronary intervention $(\mathrm{PCl})$, the corresponding numbers were from $87 \%$ (CL 95\% 86-88\%) to $91 \%$ (CL 95\% 90-92\%) and from $49 \%$ (CL $95 \% 47-50 \%$ ) to $52 \%$ (CL 95\% 51-54\%), respectively. Non-PCI patients had a higher cardiovascular risk compared with $\mathrm{PCl}$ patients. Among PCI patients, age $>75$ years, atrial fibrillation, diabetes and peripheral arterial disease were associated with a higher risk of treatment breaks for DAPT. Among patients without PCl, ticagrelor treatment was associated with an increased risk of treatment breaks during the first 12 months compared with clopidogrel treatment.

Conclusions: From 2009 to 2012, there was an increase in the proportion of patients with MI receiving DAPT, and a longer duration of DAPT. Still, a large proportion of patients without $\mathrm{PCl}$ are discharged either without DAPT or with a short DAPT duration. These findings may indicate the need for more careful attention to DAPT for patients with MI not undergoing $\mathrm{PCl}$ in Denmark.

\section{Strengths and limitations of this study}

- Our study describes dual antiplatelet treatment in Danish patients after myocardial infarction during 2009-2012, making use of the nationwide and complete health registers that may be linked at individual level by means of the unique personal identification system covering all Danish citizens.

- The registry data available for our study are collected for administrative purposes, thereby reducing potential sources of bias otherwise introduced by selection of particular hospitals or healthcare insurance systems.

- Even though coding errors cannot be ruled out in the registry data, previous studies have demonstrated high levels of sensitivity and specificity for cardiovascular outcomes in the Danish health registers.

- Our study is limited by not including information on unstable angina, ST segment elevation myocardial infarction (STEMI), non-STEMI, blood pressure, smoking habits, lipid profiles and socioeconomic status.

\section{INTRODUCTION}

Platelet activation and subsequent aggregation represent the key targets in the management of acute coronary syndromes (ACS) to prevent recurrent events. However, the incidence of ACS has declined over time, supporting the notion that contemporary treatment effectively improves outcomes after a myocardial infarction (MI). ${ }^{1-4}$ European guidelines recommend initiation of dual antiplatelet therapy (DAPT) with low-dose acetyl salicylic acid (ASA) and a $\mathrm{P}_{2} \mathrm{Y}_{12}$ antagonist to reduce the risk of acute ischaemic complications and recurrent atherothrombotic events. ${ }^{5}$ This treatment is recommended 
for up to 12 months in patients with ACS, irrespective of whether the patient undergoes revascularisation with percutaneous coronary intervention (PCI) or not. ${ }^{56}$

Previously, a nationwide Danish study described initiation and persistence patterns for DAPT with clopidogrel and ASA after MI in the years 2000-2005. ${ }^{7}$ The study showed a high persistence with clopidogrel treatment among PCI-treated patients as compared with non-PCI patients, and a lower degree of clopidogrel use among women and patients admitted to local hospitals. ${ }^{7}$

New $\mathrm{P}_{2} \mathrm{Y}_{12}$ antagonists have recently been introduced in the treatment of patients with ACS; prasugrel received European Medicines Agency (EMA) approval in 2009 and ticagrelor in 2011. Ticagrelor, co-administered with ASA, is indicated for patients with ACS, including patients managed medically, and those who are managed with PCI or coronary artery bypass grafting $(\mathrm{CABG}){ }^{8}$ Prasugrel, co-administered with ASA, is indicated for patients with ACS undergoing PCI. ${ }^{9}$

In 2011, ticagrelor was recommended as first-line treatment in the national Danish ACS guidelines across subdiagnoses. ${ }^{10}$ How these new multiple DAPT options are used in contemporary clinical practice in Denmark and how guideline recommendations are implemented are not known. Also, since the indication for the different $\mathrm{P}^{2} \mathrm{Y}_{12}$ antagonists differs, it is likely that the populations treated with the respective $\mathrm{P}_{2} \mathrm{Y}_{12}$ antagonists diverge with respect to their baseline characteristics. To the best of our knowledge, this has not been investigated in a large-scale study. Furthermore, it is of clinical relevance to describe treatment persistence and patient characteristics that are associated with reduced persistence.

The aim of this study was to describe the DAPT pattern in Danish patients with MI during 2009-2012, with the focus on comparing treatment in 2009 and 2012, that is, before and after the introduction of prasugrel and ticagrelor, by combining data from nationwide registries on hospital admissions, prescription drug use and date of mortality.

\section{METHODS}

\section{Data sources}

Data were obtained from Danish nationwide compulsory registries on hospital admissions and prescribed drugs. Since virtually all medical care in Denmark is provided by the national health authorities, these data sources allow true population-based studies with national coverage and high levels of completeness. ${ }^{11}$

The Danish National Prescription Registry ${ }^{12}$ contains data on all prescribed drugs dispensed from Danish community pharmacies since 1995. Prescription data include type of drug, date of dispensing and quantity and are categorised according to the Anatomic Therapeutic Chemical (ATC) index. ${ }^{13}$ Drug expenses are partially reimbursed by the Danish healthcare authorities.

The Danish National Patient Registry contains data on all somatic hospitalisations in Denmark since 1977 and on outpatient visits since $1995 .{ }^{14}$ Hospital discharge and outpatient contact diagnoses are coded according to the International Classification of Diseases (ICD-10) from 1994 onwards.

All data sources were linked by means of the personal identification number, a unique identifier encoding gender and date of birth, assigned by the Danish Civil Registration System to all Danish residents since 1968. ${ }^{15}$ The Civil Registration System contains continuously updated data on address, date of death and migration to and from Denmark. All record linkages were performed by Statistics Denmark.

\section{Study design and study population}

Patients who experienced a first time ever hospital admission related to acute MI within the observation period 1 January 2009 to 31 December 2012 were eligible for inclusion. An MI event was defined as having an admission with a primary or secondary diagnosis ICD-10 code of I21. Patients with a diagnosis of unstable angina pectoris (ICD-10 code I20.0) were not included. Further, subclassification into ST segment elevation MI (STEMI) and non-STEMI (NSTEMI) was not performed since ICD-10 coding specification at this level has not been validated.

An MI episode may present as a sequence of admissions to more than one hospital department and was defined as one admission if the interval was not more than 1 day between discharge from one hospital and admission date at the next hospital. Only the first episode for each individual within the observation period was included. We also required that individuals were discharged alive. Patients had to be Danish residents with a Danish permanent address at the time of admission.

The study was approved by the Danish Data Protection Agency. According to Danish law, ethical approval is not required for registry-based studies. ${ }^{16}$

\section{ANALYSIS}

All individuals were classified according to whether they had been dispensed DAPT or not. The use of DAPT was analysed among individuals experiencing MI in 2009 and 2012, respectively. All analyses were stratified by type of DAPT, study year and whether or not the patient underwent PCI in relation to the index event.

\section{Baseline characteristics of participants initiating DAPT following MI}

Individuals were described regarding age and gender, the type of hospital at index event, procedures during index event, previous diagnoses and dispensed drugs at the time of admission.

1. Classification according to admission by type of hospital according to degree of cardiological expertise available was: local hospital, hospital without catheterisation laboratory (level 1); main regional hospital, 
hospital with catheterisation laboratory (level 2); tertiary cardiac hospital, university hospital with catheterisation laboratory (level 3).

2. Procedures during index event included angiography (UXAC85), PCI (procedure code FNG) and CABG (procedure code FNA-FNE). We included CABG performed up to 30 days after discharge. Throughout the study period, procedures were coded according to the Nordic classification scheme. ${ }^{17}$

3. Previous diagnoses (other than those related to MI) registered in the Patient Registry up to 5 years prior to the admission for index MI were included. For a full list of diagnoses and definitions, see online supplementary appendix A.

4. Drug use was defined as having filled a prescription for the given drug according to the Prescription Registry within 180 days prior to the index admission and up to 30 days following discharge. For a full list of drugs included, see online supplementary appen$\operatorname{dix} B$.

\section{Persistence to DAPT following treatment initiation}

DAPTs were defined as the concomitant use of low-dose ASA and a $\mathrm{P}_{2} \mathrm{Y}_{12}$ antagonist, and were further subcategorised by the specific $\mathrm{P}_{2} \mathrm{Y}_{12}$ antagonists. The main drugs examined were the three $\mathrm{P}_{2} \mathrm{Y}_{12}$ antagonists currently available in Denmark, that is, clopidogrel (ATC B01AC04), prasugrel (B01AC22) and ticagrelor (B01AC24), as well as low-dose ASA (B01AC06 or N02BA01). For all four drugs, use was defined as having filled a prescription for the given drug within 90 days prior to the admission to 30 days after the admission. Individuals filling prescriptions for two different $\mathrm{P}_{2} \mathrm{Y}_{12}$ antagonists within this interval were classified according to the last prescription filled. Individuals failing to fill a prescription for either a $\mathrm{P}_{2} \mathrm{Y}_{12}$ antagonist or ASA within 30 days after index MI were classified as not using DAPT.

Persistence with treatment was analysed during a period of 365 days following the index MI using the "proportion of patients covered' (PPC) method. ${ }^{18}$ In brief, all participants were followed starting 30 days after discharge from the index event. Over time, we estimated the proportion of all participants still alive and not migrated and using the same $\mathrm{P}_{2} \mathrm{Y}_{12}$ antagonist as at discharge. A participant was considered a current user of a given $\mathrm{P}_{2} \mathrm{Y}_{12}$ antagonist from the day of filling a prescription for that drug and for a number of days corresponding to either the number of tablets for clopidogrel and prasugrel (used once daily) or half the number of tablets for ticagrelor (used twice daily). Finally, a 30-day grace period was added to the estimated duration to account for minor non-compliance and irregular prescription refills. A sensitivity analysis with a grace period of 60 days was also performed. An individual could be regarded as dropped out of treatment at one point in time and later be reclassified as a current user on filling a new prescription. In the Cox regression analysis for having a treatment break larger than the 30-day grace period, the type of DAPT treatment, age and gender, type of treating hospital department and selected comorbidities were chosen as covariates.

\section{Frequency of switch between different DAPT regimens}

To estimate switch patterns, we estimated the proportion of all participants who within the first year following discharge filled a $\mathrm{P}_{2} \mathrm{Y}_{12}$ antagonist other than the one they first used following discharge. The observation period for this analysis started 30 days after discharge with the index admission of MI.

\section{Statistical programme}

All calculations were performed using STATA Release V.13.0 (StataCorp, College Station, Texas, USA).

\section{RESULTS}

Overall, 97\% (28 449 patients) of all patients admitted to the hospital with a first-time MI during 2009-2012 were alive 30 days after discharge and included in this study. The baseline characteristics for the years 2009 and 2012 are shown in tables 1 and 2. Baseline characteristics for the total material as well as for the years 2010 and 2011 are contained in online supplementary tables S1-S3, respectively.

\section{Patient characteristics 2009}

Of the patients with first-time MI (median age 69 years (IQR 59-79 years); 36\% women), 73\% underwent angiography and 53\% PCI, and a majority of patients (67\%) were discharged with DAPT (table 1 and figure 1). A larger proportion of patients with PCI were discharged with DAPT $(87 \%)$ compared with the patients without PCI $(51 \%)$. The PCI patients were younger and more frequently men than the non-PCI patients. A majority of these patients received ASA, $\beta$-blockers, ACE inhibitors and statins at discharge, which is in line with guideline recommendations. Among the non-PCI patients, a considerably larger proportion underwent $\mathrm{CABG}$, had a diagnosis of atrial fibrillation and/or had a history of major bleedings compared with the PCI patients. Notably, a larger proportion of non-PCI patients were discharged without $\beta$-blockers, ACE inhibitors and statins.

\section{Patient characteristics 2012}

The median age of patients with first-time MI was 69 years (IQR 58-78 years), and 36\% were women (table 2). Overall, 79\% underwent angiography and $55 \%$ PCI, and the majority $(73 \%)$ were discharged with DAPT (table 2 and figure 1). Still, a large proportion $(49 \%)$ of the non-PCI patients were discharged without DAPT and other guideline recommended drug therapies compared with the PCI patients. In general, marked differences in patient characteristic were observed to be dependent on the choice of $\mathrm{P}_{2} \mathrm{Y}_{12}$ antagonist used in the DAPT regimens. Patients treated with prasugrel were 


\begin{tabular}{|c|c|c|c|c|c|c|c|c|c|c|}
\hline & \multicolumn{5}{|c|}{ Patients with $\mathrm{PCl}(\mathrm{N}=3576,50 \%)$} & \multicolumn{5}{|c|}{ Patients without $\mathrm{PCl}(\mathrm{N}=3528,50 \%)$} \\
\hline & $\begin{array}{l}\text { All patients } \\
\mathrm{n}=3576\end{array}$ & $\begin{array}{l}\text { Clopidogrel } \\
\mathrm{n}=3087\end{array}$ & $\begin{array}{l}\text { Ticagrelor } \\
\mathrm{n}=\mathbf{0}\end{array}$ & $\begin{array}{l}\text { Prasugrel } \\
n=13\end{array}$ & $\begin{array}{l}\text { No DAPT } \\
\mathrm{n}=476\end{array}$ & $\begin{array}{l}\text { All patients } \\
n=3528\end{array}$ & $\begin{array}{l}\text { Clopidogrel } \\
n=1712\end{array}$ & $\begin{array}{l}\text { Ticagrelor } \\
\mathrm{n}=0\end{array}$ & $\begin{array}{l}\text { Prasugrel } \\
n=1\end{array}$ & $\begin{array}{l}\text { No DAPT } \\
\mathrm{n}=1815\end{array}$ \\
\hline Age (median (IQR)) & $64(55-73)$ & $64(55-73)$ & - & $57(50-64)$ & $68(59-75)$ & $74(64-83)$ & $74(64-83)$ & - & $49(49-49)$ & $74(63-84)$ \\
\hline Males & 2643 (73.9\%) & 2289 (74.1\%) & $0(\%)$ & $8(61.5 \%)$ & $346(72.7 \%)$ & $1951(55.3 \%)$ & $956(55.8 \%)$ & $0(\%)$ & $0(0.0 \%)$ & 995 (54.8\%) \\
\hline \multicolumn{11}{|l|}{ Type of hospital (at index Ml event) } \\
\hline Local hospital & $946(26.5 \%)$ & $805(26.1 \%)$ & $0(\%)$ & $5(38.5 \%)$ & $136(28.6 \%)$ & 1440 (40.8\%) & $659(38.5 \%)$ & $0(\%)$ & $<5$ & $780(43.0 \%)$ \\
\hline Main regional hospital & $1149(32.1 \%)$ & 1005 (32.6\%) & $0(\%)$ & $5(38.5 \%)$ & $139(29.2 \%)$ & $1150(32.6 \%)$ & $616(36.0 \%)$ & $0(\%)$ & $0(0.0 \%)$ & $534(29.4 \%)$ \\
\hline Tertiary cardiac hospital & $1481(41.4 \%)$ & $1277(41.4 \%)$ & $0(\%)$ & $<5$ & $201(42.2 \%)$ & $938(26.6 \%)$ & $437(25.5 \%)$ & $0(\%)$ & $0(0.0 \%)$ & $501(27.6 \%)$ \\
\hline \multicolumn{11}{|l|}{ Procedures (at index event) } \\
\hline CABG & $80(2.2 \%)$ & $47(1.5 \%)$ & $0(\%)$ & $0(0.0 \%)$ & $33(6.9 \%)$ & $412(11.7 \%)$ & $197(11.5 \%)$ & $0(\%)$ & $0(0.0 \%)$ & $215(11.8 \%)$ \\
\hline Angiography & 3535 (98.9\%) & 3055 (99.0\%) & $0(\%)$ & $13(100.0 \%)$ & 467 (98.1\%) & $1761(49.9 \%)$ & $932(54.4 \%)$ & $0(\%)$ & $<5$ & $828(45.6 \%)$ \\
\hline \multicolumn{11}{|l|}{ Previous diagnoses } \\
\hline Heart failure & $100(2.8 \%)$ & $71(2.3 \%)$ & $0(\%)$ & $0(0.0 \%)$ & $29(6.1 \%)$ & $343(9.7 \%)$ & $148(8.6 \%)$ & $0(\%)$ & $0(0.0 \%)$ & $195(10.7 \%)$ \\
\hline Ischaemic heart disease & $256(7.2 \%)$ & $181(5.9 \%)$ & $0(\%)$ & $<5$ & $74(15.5 \%)$ & $488(13.8 \%)$ & $199(11.6 \%)$ & $0(\%)$ & $0(0.0 \%)$ & $289(15.9 \%)$ \\
\hline Unstable angina & $55(1.5 \%)$ & $43(1.4 \%)$ & $0(\%)$ & $0(0.0 \%)$ & $12(2.5 \%)$ & $107(3.0 \%)$ & $50(2.9 \%)$ & $0(\%)$ & $0(0.0 \%)$ & $57(3.1 \%)$ \\
\hline Peripheral arterial disease & $94(2.6 \%)$ & $67(2.2 \%)$ & $0(\%)$ & $0(0.0 \%)$ & $27(5.7 \%)$ & $246(7.0 \%)$ & $118(6.9 \%)$ & $0(\%)$ & $0(0.0 \%)$ & $128(7.1 \%)$ \\
\hline Stroke total & $132(3.7 \%)$ & $98(3.2 \%)$ & $0(\%)$ & $<5$ & $33(6.9 \%)$ & $338(9.6 \%)$ & $160(9.3 \%)$ & $0(\%)$ & $0(0.0 \%)$ & $178(9.8 \%)$ \\
\hline Non-ischaemic stroke & $<5$ & $<5$ & $0(\%)$ & $0(0.0 \%)$ & $<5$ & $19(0.5 \%)$ & 7 (0.4\%) & $0(\%)$ & $0(0.0 \%)$ & $12(0.7 \%)$ \\
\hline Ischaemic stroke & $130(3.6 \%)$ & $96(3.1 \%)$ & $0(\%)$ & $<5$ & $33(6.9 \%)$ & $328(9.3 \%)$ & $155(9.1 \%)$ & $0(\%)$ & $0(0.0 \%)$ & $173(9.5 \%)$ \\
\hline Atrial fibrillation & $125(3.5 \%)$ & $93(3.0 \%)$ & $0(\%)$ & $0(0.0 \%)$ & $32(6.7 \%)$ & $375(10.6 \%)$ & $132(7.7 \%)$ & $0(\%)$ & $0(0.0 \%)$ & $243(13.4 \%)$ \\
\hline Chronic renal dysfunction & $16(0.4 \%)$ & $11(0.4 \%)$ & $0(\%)$ & $0(0.0 \%)$ & $5(1.1 \%)$ & $43(1.2 \%)$ & $18(1.1 \%)$ & $0(\%)$ & $0(0.0 \%)$ & $25(1.4 \%)$ \\
\hline Diabetes mellitus & $396(11.1 \%)$ & $327(10.6 \%)$ & $0(\%)$ & $<5$ & $68(14.3 \%)$ & $625(17.7 \%)$ & $304(17.8 \%)$ & $0(\%)$ & $0(0.0 \%)$ & $321(17.7 \%)$ \\
\hline Major bleeding & $93(2.6 \%)$ & $75(2.4 \%)$ & $0(\%)$ & $0(0.0 \%)$ & $18(3.8 \%)$ & $189(5.4 \%)$ & $67(3.9 \%)$ & $0(\%)$ & $0(0.0 \%)$ & $122(6.7 \%)$ \\
\hline Liver disease & $<5$ & $<5$ & $0(\%)$ & $0(0.0 \%)$ & $0(0.0 \%)$ & $5(0.1 \%)$ & $<5$ & $0(\%)$ & $0(0.0 \%)$ & $<5$ \\
\hline Coagulation disorders & $9(0.3 \%)$ & $8(0.3 \%)$ & $0(\%)$ & $0(0.0 \%)$ & $<5$ & $21(0.6 \%)$ & $<5$ & $0(\%)$ & $0(0.0 \%)$ & $17(0.9 \%)$ \\
\hline Cancer & $193(5.4 \%)$ & $158(5.1 \%)$ & $0(\%)$ & $<5$ & $34(7.1 \%)$ & $333(9.4 \%)$ & $146(8.5 \%)$ & $0(\%)$ & $0(0.0 \%)$ & $187(10.3 \%)$ \\
\hline \multicolumn{11}{|l|}{ Drug use at discharge } \\
\hline Total number of drugs (median (IQR)) & $3(1-6)$ & $3(1-6)$ & - & $4(0-8)$ & $4(2-8)$ & $6(3-10)$ & $6(3-10)$ & - & $13(13-13)$ & $7(3-11)$ \\
\hline ACE inhbitors and ARB & 1948 (54.5\%) & $1678(54.4 \%)$ & $0(\%)$ & $7(53.8 \%)$ & $263(55.3 \%)$ & $1984(56.2 \%)$ & $992(57.9 \%)$ & $0(\%)$ & $0(0.0 \%)$ & $992(54.7 \%)$ \\
\hline Acetyl salicylic acid & 3379 (94.5\%) & 3087 (100.0\%) & $0(\%)$ & $13(100.0 \%)$ & $279(58.6 \%)$ & 2962 (84.0\%) & $1712(100.0 \%)$ & $0(\%)$ & $<5$ & $1249(68.8 \%)$ \\
\hline$\beta$-blocker & 3170 (88.6\%) & 2760 (89.4\%) & $0(\%)$ & $12(92.3 \%)$ & $398(83.6 \%)$ & 2537 (71.9\%) & $1389(81.1 \%)$ & $0(\%)$ & $0(0.0 \%)$ & $1148(63.3 \%)$ \\
\hline Calcium-channel blocker & 749 (20.9\%) & $621(20.1 \%)$ & $0(\%)$ & $<5$ & $127(26.7 \%)$ & 1027 (29.1\%) & $474(27.7 \%)$ & $0(\%)$ & $<5$ & $552(30.4 \%)$ \\
\hline Oral antidiabetics and insulin & $369(10.3 \%)$ & $308(10.0 \%)$ & $0(\%)$ & $<5$ & $60(12.6 \%)$ & $562(15.9 \%)$ & $277(16.2 \%)$ & $0(\%)$ & $0(0.0 \%)$ & 285 (15.7\%) \\
\hline Proton pump inhibitors & $916(25.6 \%)$ & $772(25.0 \%)$ & $0(\%)$ & $<5$ & $140(29.4 \%)$ & $1302(36.9 \%)$ & $579(33.8 \%)$ & $0(\%)$ & $<5$ & $722(39.8 \%)$ \\
\hline Statins & 3379 (94.5\%) & 2954 (95.7\%) & $0(\%)$ & $12(92.3 \%)$ & $413(86.8 \%)$ & $2523(71.5 \%)$ & $1427(83.4 \%)$ & $0(\%)$ & $<5$ & $1095(60.3 \%)$ \\
\hline Anticoagulant & $224(6.3 \%)$ & $162(5.2 \%)$ & $0(\%)$ & $<5$ & $61(12.8 \%)$ & $415(11.8 \%)$ & $113(6.6 \%)$ & $0(\%)$ & $0(0.0 \%)$ & $302(16.6 \%)$ \\
\hline NSAIDs & $613(17.1 \%)$ & $531(17.2 \%)$ & $0(\%)$ & $5(38.5 \%)$ & 77 (16.2\%) & $606(17.2 \%)$ & $280(16.4 \%)$ & $0(\%)$ & $0(0.0 \%)$ & $326(18.0 \%)$ \\
\hline \multicolumn{11}{|c|}{ Time until $\mathrm{P}_{2} \mathrm{Y}_{12}$ antagonist prescription claimed } \\
\hline Prior to $\mathrm{Ml}$ & $122(3.4 \%)$ & 103 (3.3\%) & $0(\%)$ & $<5$ & $18(3.8 \%)$ & 167 (4.7\%) & 125 (7.3\%) & $0(\%)$ & $0(0.0 \%)$ & $42(2.3 \%)$ \\
\hline $1-7$ days & 3033 (84.8\%) & 2825 (91.5\%) & $0(\%)$ & $11(84.6 \%)$ & 197 (41.4\%) & 1600 (45.4\%) & 1438 (84.0\%) & $0(\%)$ & $<5$ & 161 (8.9\%) \\
\hline $8-14$ days & $52(1.5 \%)$ & $48(1.6 \%)$ & $0(\%)$ & $0(0.0 \%)$ & $<5$ & $69(2.0 \%)$ & $65(3.8 \%)$ & $0(\%)$ & $0(0.0 \%)$ & $<5$ \\
\hline $15-30$ days & $120(3.4 \%)$ & $111(3.6 \%)$ & $0(\%)$ & $<5$ & $8(1.7 \%)$ & $91(2.6 \%)$ & $84(4.9 \%)$ & $0(\%)$ & $0(0.0 \%)$ & $7(0.4 \%)$ \\
\hline No prescription & 249 (7.0\%) & 0 (0.0\%) & $0(\%)$ & $0(0.0 \%)$ & 249 (52.3\%) & 1601 (45.4\%) & 0 (0.0\%) & $0(\%)$ & $0(0.0 \%)$ & 1601 (88.2\%) \\
\hline
\end{tabular}

Numbers in parentheses are percentages of total number of patients in the group; local hospital, hospital without catheterisation laboratory; main regional hospital, hospital with catheterisation laboratory; tertiary cardiac hospital, university hospital with catheterisation laboratory.

ARB, angiotensin receptor blocker; CABG, coronary artery bypass graft; DAPT, dual antiplatelet therapy; MI, myocardial infarction; NSAIDs, non-steroidal anti-inflammatory drugs; PCI,

percutaneous coronary intervention. 


\begin{tabular}{|c|c|c|c|c|c|c|c|c|c|c|}
\hline & \multicolumn{5}{|c|}{ Patients with $\mathrm{PCl}(\mathrm{n}=3852,55 \%)$} & \multicolumn{5}{|c|}{ Non-PCI patients ( $n=3164,45 \%)$} \\
\hline & $\begin{array}{l}\text { All patients } \\
\mathrm{n}=3852\end{array}$ & $\begin{array}{l}\text { Clopidogrel } \\
\mathrm{n}=724\end{array}$ & $\begin{array}{l}\text { Ticagrelor } \\
\mathrm{n}=2238\end{array}$ & $\begin{array}{l}\text { Prasugrel } \\
n=531\end{array}$ & $\begin{array}{l}\text { No DAPT } \\
\mathrm{n}=359\end{array}$ & $\begin{array}{l}\text { All patients } \\
\mathrm{n}=3164\end{array}$ & $\begin{array}{l}\text { Clopidogrel } \\
\mathrm{n}=679\end{array}$ & $\begin{array}{l}\text { Ticagrelor } \\
\mathrm{n}=921\end{array}$ & $\begin{array}{l}\text { Prasugrel } \\
\mathrm{n}=26\end{array}$ & $\begin{array}{l}\text { No DAPT } \\
\mathrm{n}=1538\end{array}$ \\
\hline Age (median (IQR)) & $65(55-74)$ & $68(58-79)$ & $65(55-74)$ & $58(51-66)$ & $69(59-77)$ & $74(63-83)$ & $77(67-86)$ & $71(61-80)$ & $52.5(48-67)$ & $75(64-84)$ \\
\hline Males & $2800(72.7)$ & $490(67.7)$ & $1615(72.2)$ & $440(82.9)$ & $255(71.0)$ & $1684(53.2)$ & $337(49.6)$ & $500(54.3)$ & $18(69.2)$ & $829(53.9)$ \\
\hline \multicolumn{11}{|l|}{ Type of hospital (at index Ml event) } \\
\hline Local hospital & 1037 (26.9) & $291(40.2)$ & $552(24.7)$ & $68(12.8)$ & $126(35.1)$ & $1285(40.6)$ & $277(40.8)$ & $318(34.5)$ & $<5$ & $686(44.6)$ \\
\hline Main regional hospital & $1579(41.0)$ & 202 (27.9) & $1021(45.6)$ & $234(44.1)$ & $122(34.0)$ & $1144(36.2)$ & $247(36.4)$ & $417(45.3)$ & $8(30.8)$ & $472(30.7)$ \\
\hline Tertiary cardiac hospital & $1236(32.1)$ & 231 (31.9) & $665(29.7)$ & $229(43.1)$ & $111(30.9)$ & 735 (23.2) & $155(22.8)$ & $186(20.2)$ & $14(53.8)$ & $380(24.7)$ \\
\hline \multicolumn{11}{|l|}{ Procedures (at index Ml event) } \\
\hline CABG & $93(2.4)$ & $40(5.5)$ & $20(0.9)$ & $<5$ & $30(8.4)$ & $453(14.3)$ & 77 (11.3) & $130(14.1)$ & $<5$ & $245(15.9)$ \\
\hline Angiography & $3790(98.4)$ & $704(97.2)$ & $2213(98.9)$ & $525(98.9)$ & $348(96.9)$ & $1740(55.0)$ & $340(50.1)$ & $649(70.5)$ & $16(61.5)$ & $735(47.8)$ \\
\hline \multicolumn{11}{|l|}{ Previous diagnoses } \\
\hline Heart failure & $83(2.2)$ & $23(3.2)$ & $34(1.5)$ & $7(1.3)$ & $19(5.3)$ & $234(7.4)$ & $67(9.9)$ & $41(4.5)$ & $0(0.0)$ & $126(8.2)$ \\
\hline Ischaemic heart disease & $210(5.5)$ & $59(8.1)$ & $90(4.0)$ & $16(3.0)$ & $45(12.5)$ & $413(13.1)$ & $108(15.9)$ & $101(11.0)$ & $<5$ & $200(13.0)$ \\
\hline Unstable angina pectoris & 46 (1.2) & $14(1.9)$ & $21(0.9)$ & $<5$ & $9(2.5)$ & $77(2.4)$ & $18(2.7)$ & $14(1.5)$ & $<5$ & $43(2.8)$ \\
\hline Peripheral arterial disease & $110(2.9)$ & $36(5.0)$ & $44(2.0)$ & $6(1.1)$ & $24(6.7)$ & $224(7.1)$ & $61(9.0)$ & $55(6.0)$ & $0(0.0)$ & $108(7.0)$ \\
\hline Stroke total & $126(3.3)$ & $36(5.0)$ & $62(2.8)$ & $<5$ & $25(7.0)$ & $257(8.1)$ & $86(12.7)$ & $40(4.3)$ & $0(0.0)$ & $131(8.5)$ \\
\hline Non-ischaemic stroke & $12(0.3)$ & $<5$ & $6(0.3)$ & $0(0.0)$ & $<5$ & $22(0.7)$ & $5(0.7)$ & $<5$ & $0(0.0)$ & $14(0.9)$ \\
\hline Ischaemic stroke & $117(3.0)$ & $33(4.6)$ & $58(2.6)$ & $<5$ & $23(6.4)$ & $243(7.7)$ & 83 (12.2) & $38(4.1)$ & $0(0.0)$ & $122(7.9)$ \\
\hline Atrial fibrillation & $150(3.9)$ & $44(6.1)$ & $56(2.5)$ & $5(0.9)$ & 45 (12.5) & $335(10.6)$ & $63(9.3)$ & $49(5.3)$ & $0(0.0)$ & $223(14.5)$ \\
\hline Chronic renal dysfunction & $30(0.8)$ & $8(1.1)$ & $11(0.5)$ & $<5$ & $10(2.8)$ & $41(1.3)$ & $11(1.6)$ & $<5$ & $<5$ & $24(1.6)$ \\
\hline Diabetes mellitus & $512(13.3)$ & $114(15.7)$ & $279(12.5)$ & $53(10.0)$ & $66(18.4)$ & $585(18.5)$ & $157(23.1)$ & $140(15.2)$ & $<5$ & $284(18.5)$ \\
\hline Major bleeding & $88(2.3)$ & $26(3.6)$ & $41(1.8)$ & 7 (1.3) & $14(3.9)$ & $155(4.9)$ & $33(4.9)$ & $33(3.6)$ & $0(0.0)$ & $89(5.8)$ \\
\hline Liver disease & $9(0.2)$ & $<5$ & $<5$ & $<5$ & $<5$ & $7(0.2)$ & $<5$ & $<5$ & $0(0.0)$ & $<5$ \\
\hline Coagulation disorders & $15(0.4)$ & $5(0.7)$ & $<5$ & $0(0.0)$ & $6(1.7)$ & $17(0.5)$ & $<5$ & $<5$ & $0(0.0)$ & $14(0.9)$ \\
\hline Cancer & $278(7.2)$ & $66(9.1)$ & $145(6.5 \%)$ & $25(4.7)$ & $42(11.7)$ & $339(10.7)$ & $63(9.3)$ & $86(9.3)$ & $0(0.0)$ & $190(12.4)$ \\
\hline \multicolumn{11}{|l|}{ Drug use at discharge } \\
\hline Total number of drugs (median (IQR)) & $3(1-6)$ & $4(1-8)$ & $3(1-6)$ & $2(0-4)$ & $5(2-8)$ & $6(3-10)$ & $7(4-11)$ & $5(2-9)$ & $4.5(1-6)$ & $7(3-11)$ \\
\hline ACE inhibitors and ARB & $2076(53.9)$ & $450(62.2)$ & $1145(51.2)$ & $268(50.5)$ & $213(59.3)$ & $1748(55.2)$ & $417(61.4)$ & $495(53.7)$ & $15(57.7)$ & $821(53.4)$ \\
\hline Acetyl salicylic acid & 3666 (95.2) & $724(100.0)$ & $2238(100.0)$ & $531(100.0)$ & $173(48.2)$ & $2568(81.2)$ & $679(100.0)$ & $921(100.0)$ & $26(100.0)$ & $942(61.2)$ \\
\hline$\beta$-blocker & $3364(87.3)$ & $621(85.8)$ & $1959(87.5)$ & $503(94.7)$ & $281(78.3)$ & $2226(70.4)$ & $517(76.1)$ & $754(81.9)$ & $20(76.9)$ & $935(60.8)$ \\
\hline Calcium-channel blocker & $877(22.8)$ & $218(30.1)$ & $463(20.7)$ & 79 (14.9) & $117(32.6)$ & $1073(33.9)$ & $247(36.4)$ & $305(33.1)$ & $12(46.2)$ & $509(33.1)$ \\
\hline Oral antidiabetics and insulin & $473(12.3)$ & $98(13.5)$ & $265(11.8)$ & $49(9.2)$ & $61(17.0)$ & $532(16.8)$ & $147(21.6)$ & $133(14.4)$ & $<5$ & $248(16.1)$ \\
\hline Proton pump inhibitors & $1195(31.0)$ & $275(38.0)$ & $680(30.4)$ & $108(20.3)$ & $132(36.8)$ & $1280(40.5)$ & $322(47.4)$ & $323(35.1)$ & $9(34.6)$ & $626(40.7)$ \\
\hline Statins & 3661 (95.0) & 672 (92.8) & $2165(96.7)$ & $524(98.7)$ & $300(83.6)$ & $2219(70.1)$ & $529(77.9)$ & $802(87.1)$ & $22(84.6)$ & $866(56.3)$ \\
\hline Anticoagulant & $266(6.9)$ & $76(10.5)$ & $94(4.2)$ & $14(2.6)$ & $82(22.8)$ & $445(14.1)$ & $65(9.6)$ & $63(6.8)$ & $0(0.0)$ & $317(20.6)$ \\
\hline NSAIDs & $624(16.2)$ & $118(16.3)$ & $367(16.4)$ & 79 (14.9) & $60(16.7)$ & $539(17.0)$ & $104(15.3)$ & $159(17.3)$ & $8(30.8)$ & $268(17.4)$ \\
\hline \multicolumn{11}{|c|}{ Time until $\mathrm{P}_{2} \mathrm{Y}_{12}$ antagonist prescription claimed } \\
\hline Prior to $\mathrm{Ml}$ & $165(4.3)$ & $48(6.6)$ & $80(3.6)$ & $16(3.0)$ & $21(5.8)$ & $228(7.2)$ & $121(17.8)$ & $45(4.9)$ & $<5$ & $61(4.0)$ \\
\hline $1-7$ days & $3472(90.1)$ & $656(90.6)$ & $2123(94.9)$ & $508(95.7)$ & $185(51.5)$ & $1543(48.8)$ & $521(76.7)$ & $833(90.4)$ & $24(92.3)$ & $165(10.7)$ \\
\hline 8-14 days & $32(0.8)$ & $8(1.1)$ & $11(0.5)$ & $7(1.3)$ & $6(1.7)$ & $46(1.5)$ & $21(3.1)$ & $21(2.3)$ & $0(0.0)$ & $<5$ \\
\hline $15-30$ days & $44(1.1)$ & $12(1.7)$ & $24(1.1)$ & $0(0.0)$ & $8(2.2)$ & $49(1.5)$ & $16(2.4)$ & $22(2.4)$ & $<5$ & $10(0.7)$ \\
\hline No prescription & $139(3.6)$ & $0(0.0)$ & $0(0.0)$ & $0(0.0)$ & 139 (38.7) & $1298(41.0)$ & $0(0.0)$ & $0(0.0)$ & $0(0.0)$ & $1298(84.4)$ \\
\hline
\end{tabular}

Tertiary cardiac hospital, university hospital with catheterisation laboratory.

Local hospital, hospital without catheterisation laboratory; main regional hospital, hospital with catheterisation laboratory.

Numbers in parentheses are percentages of total number of patients in the group.ARB, angiotensin receptor blocker; CABG, coronary artery bypass graft; DAPT, dual antiplatelet therapy; MI,

myocardial infarction; NSAIDs, non-steroidal anti-inflammatory drugs; PCI, percutaneous coronary intervention. 
PCl-treated patients

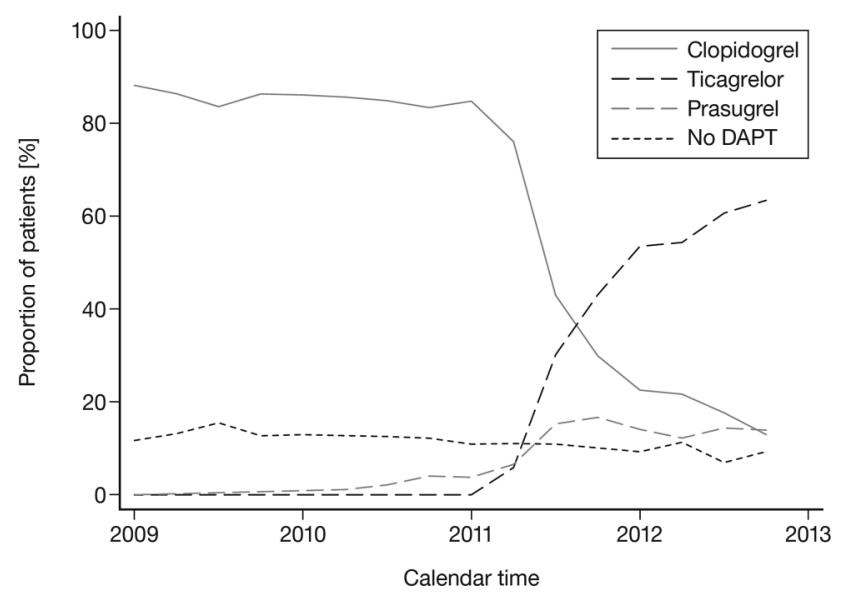

Non-PCl-treated patients

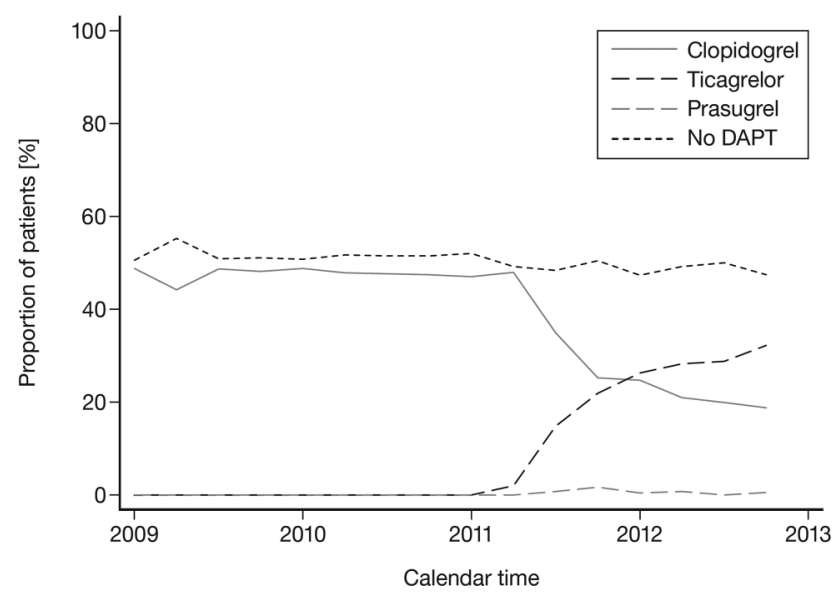

Figure 1 Proportion of patients with first-time Ml discharged alive with or without $\mathrm{PCl}$ and prescribed different types of DAPT or no DAPT 2009-2012. DAPT, dual antiplatelet therapy; MI, myocardial infarction; $\mathrm{PCl}$, percutaneous coronary intervention.

11 years younger (median), more commonly men and the majority underwent PCI (84\%) compared with the total MI patient population. Most of the prasugreltreated patients were either managed at a main regional hospital or at a university hospital with a catheterisation laboratory.

The proportion of patients prescribed DAPT with ticagrelor increased quickly after its introduction in 2011. By the end of 2012, ticagrelor was the most common $\mathrm{P}_{12} \mathrm{Y}_{12}$ antagonist in patients with and without PCI (figure 1). More patients in the ticagrelor group underwent PCI $(71 \%)$ compared with clopidogrel-treated patients $(52 \%)$. Ticagrelor-treated patients were 7 years younger and more commonly men. Patients treated with clopidogrel had in general a more severe disease burden at baseline, with additional diagnoses of heart failure, stroke or atrial fibrillation compared with the other DAPT-treated patients (table 2).

In 2012, the proportion of patients discharged with DAPT was $6 \%$ higher compared with that in 2009. The
non-DAPT-treated patients were older $(+9$ years, median age difference) and more commonly women $(+20 \%$ difference) compared with the DAPT-treated patients. Among non-DAPT-treated patients, invasive treatment within 30 days from admission was received by relatively few patients; $57 \%$ underwent angiography and 19\% PCI. A larger proportion of non-DAPT-treated patients underwent $\mathrm{CABG}$, were diagnosed with atrial fibrillation and were treated with warfarin or new oral anticoagulants (NOAC) compared with the DAPT-treated patients. More patients had a prior diagnosis of heart failure, cancer and/or a history of major bleeds or coagulation disorders.

A smaller proportion of the non-DAPT-treated patients received ACE inhibitors/angiotensin receptor blockers (ARBs), $\beta$-blockers and statins at discharge compared with DAPT-treated patients. Of these, $49 \%$ received ASA as monotherapy, and $13 \%$ and $10 \%$ of non-DAPT-treated patients were treated with clopidogrel or ticagrelor, respectively, as monotherapies.

\section{Medical history-related predictors of DAPT persistence}

Overall, persistence was very high among patients initiated on DAPT ( figure 2).

Within the first year post-MI in 2012, $6 \%$ of the prasugrel-treated patients were switched to another $\mathrm{P}_{2} \mathrm{Y}_{12}$ antagonist; $11 \%$ from the ticagrelor group and 3\% from the clopidogrel group (table 3).

Patients undergoing PCI had a longer DAPT duration overall compared with patients not undergoing PCI, and age $>75$ years and diagnosis of atrial fibrillation, diabetes and peripheral arterial disease were associated with a higher risk of treatment breaks (table 4). Furthermore, there was a trend towards increased risk for treatment breaks for PCI patients with heart failure and stroke. For patients not undergoing PCI, we did not observe any association between any major baseline diseases and risk for treatment breaks.

Among PCI patients, treatment with prasugrel or ticagrelor compared with clopidogrel was associated with an increased risk of a 30-day treatment break within 365 days after MI (table 4). However, this risk was not present when extending the grace period to 60 days (data not shown).

For non-PCI patients, ticagrelor compared with clopidogrel treatment was associated with an increased risk of having a 30-day treatment break. This finding was also present when expanding the grace period to 60 days, during which $11 \%$ of these patients were switched to clopidogrel after a median of 107 days.

\section{DISCUSSION}

This nationwide observational study showed changes in the treatment of patients with MI in Denmark from 2009 to 2012. In 2012, more patients are referred to coronary angiography and PCI, and a larger proportion of patients are discharged with DAPT compared to 2009. However, 

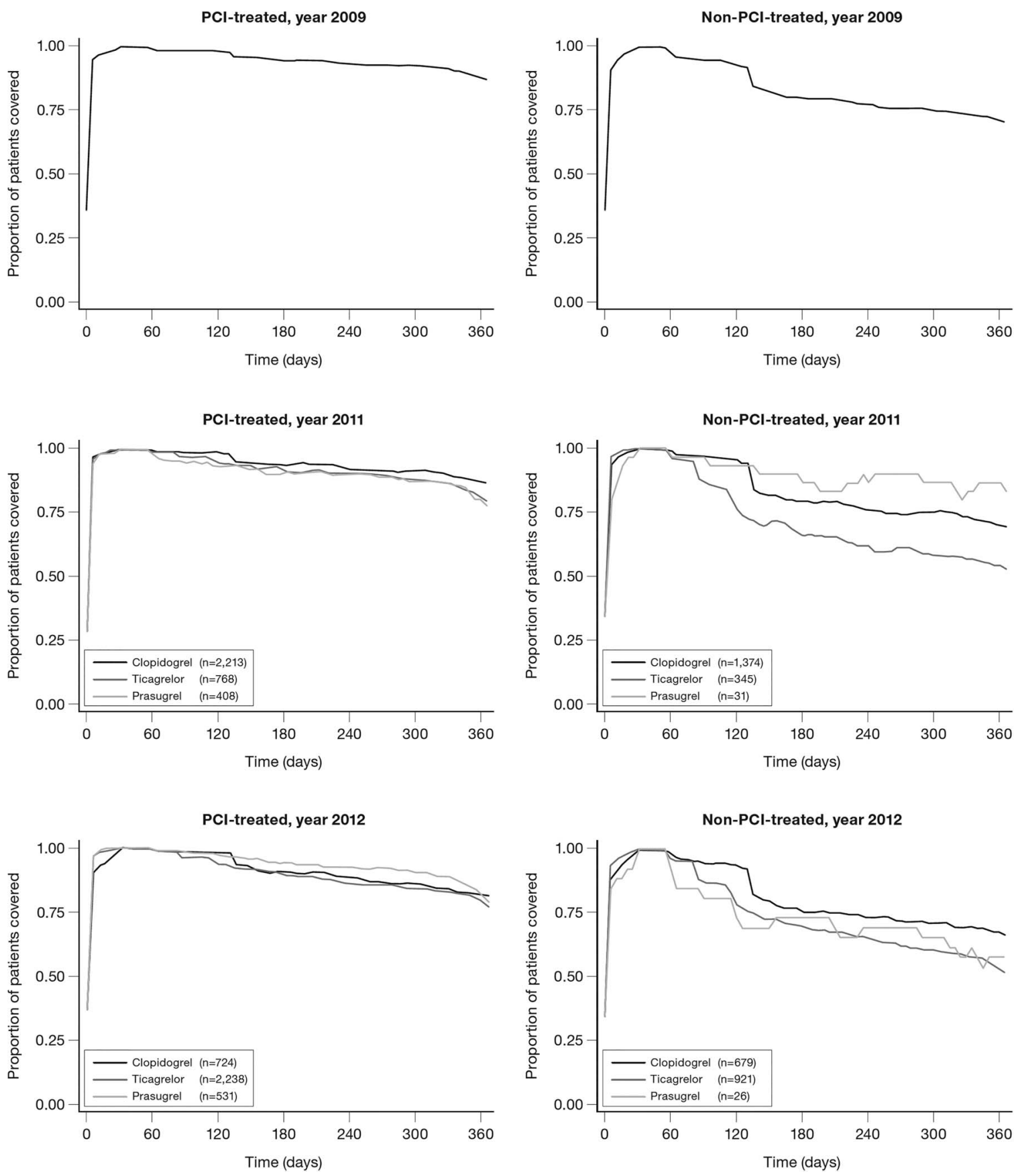

Figure 2 Persistence with different dual antiplatelet therapy in patients with first-time Ml with or without PCl 2009-2012. MI, myocardial infarction; $\mathrm{PCl}$, percutaneous coronary intervention.

non-PCI patients were, to a large extent, discharged without DAPT, or received shorter duration of DAPT treatment as compared with PCI patients. Among PCI patients, age $>75$ years, atrial fibrillation, diabetes and peripheral arterial disease were all associated with a higher risk of treatment breaks, which might indicate a risk-treatment mismatch, as these patients have a higher risk of recurrent events and might benefit from longer DAPT duration. During the observation period, the DAPT pattern shifted from merely clopidogrel treatment to more selective treatments with clopidogrel, prasugrel and ticagrelor for patient populations with varying characteristics.

\section{Interpretation with reference to other studies}

The underlying medical treatment of patients with $\mathrm{MI}$ in Denmark, with more patients undergoing angiography and PCI over time, followed the same trend as seen both in earlier observations in Denmark and studies from other countries. ${ }^{19-21}$

To the best of our knowledge, national-level data describing patient selection for different DAPT regimens and persistence with treatment in unselected populations are scarce. Publications based on data from cardiovascular quality registers, actively recruiting or selecting patients, report an overall DAPT usage for discharged 

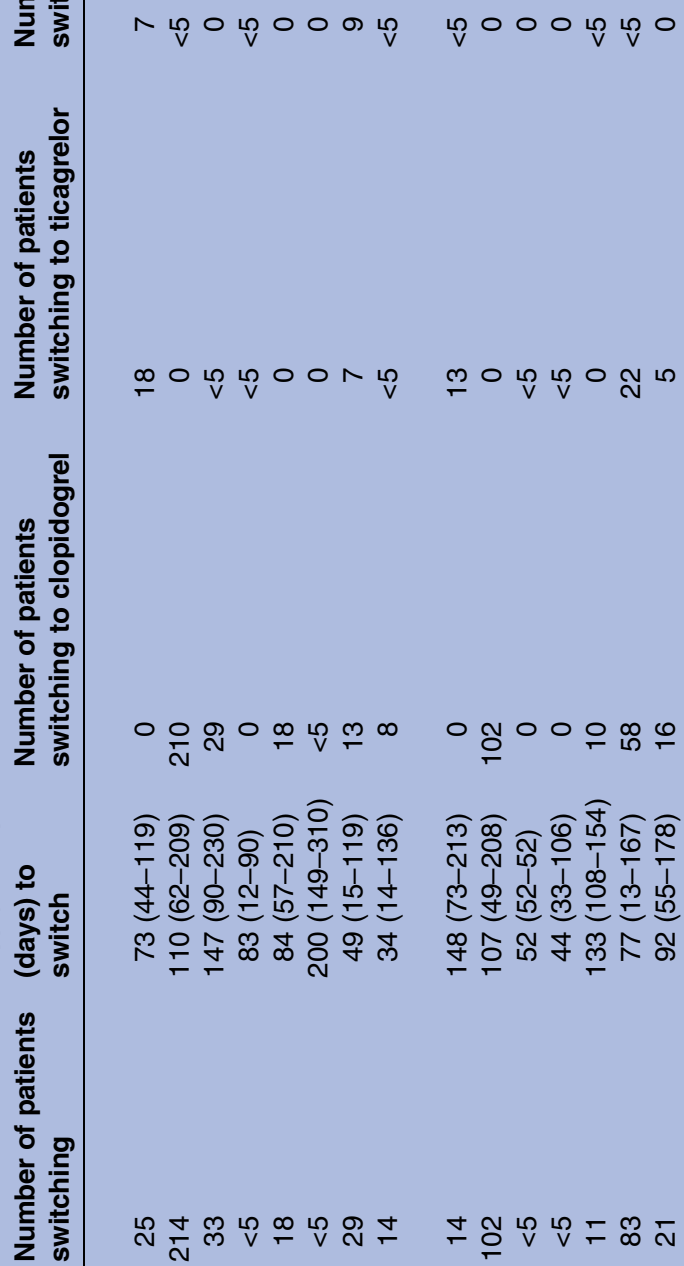

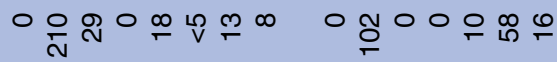

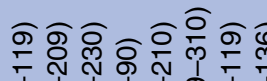

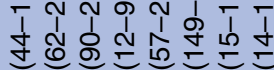

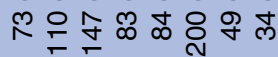

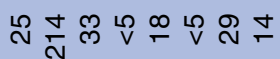

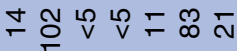

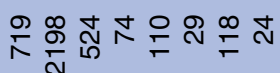

ஜூ
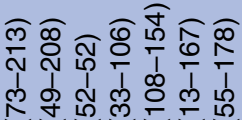

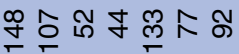

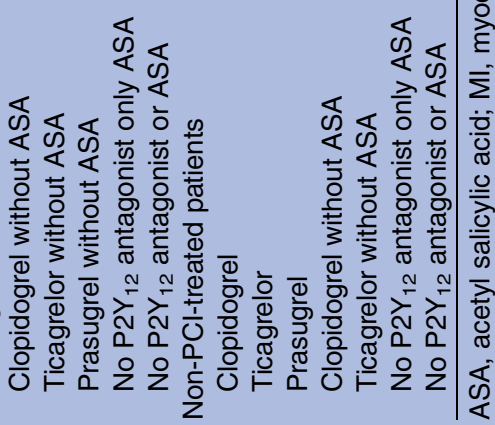

patients with ACS in the range of $\sim 60-80 \%$ depending on the observation period and the type of ACS event included. $^{21-25} \mathrm{~A}$ recent Swedish nationwide study on patients with MI, which may be considered comparable to the present nationwide data, reported a DAPT usage of $69 \%$ for patients discharged with MI in 2000-2011. ${ }^{26}$

A previous similar Danish study, including all patients with MI between 2000 and 2005, reported an increasing use of DAPT during the observation period. ${ }^{7}$ However, for non-PCI patients, there was a substantial underuse, especially among women and patients admitted to local hospitals. Although the observation period in this study is more recent, many of these patients are still discharged without DAPT, although there is a markedly increased use of DAPT in these patient groups.

The observed overall adherence to DAPT (figure 2), with more than $75 \%$ of patients completing more than 11 months of treatment, is noteworthy and comparable to what has been observed in randomised controlled trials. ${ }^{27} 28$

\section{Medical history-related predictors of DAPT persistence}

The non-DAPT-treated patients are of special interest, as in this study they form a considerable proportion of patients discharged with first-time MI (27\% in 2012), despite the decline in the relative number of patients discharged without DAPT during the observation period (figure 2). A large proportion of these patients received oral antiplatelet monotherapy with ASA or with prasugrel, ticagrelor or clopidogrel. Furthermore, there was a marked difference between patients who underwent PCI versus those who did not; a larger proportion of non-PCI patients were discharged without DAPT (in 2012: $9 \%$ vs $49 \%$, respectively). In addition, non-PCI patients had a shorter DAPT treatment duration in general, were on average 10 years older and with a large proportion having a risk profile with atrial fibrillation and history of bleedings where a shorter DAPT duration or no DAPT treatment may be appropriate. However, many of these patients had a high cardiovascular disease risk profile at baseline, suggesting a potential benefit of a longer DAPT treatment duration and more frequent use of $\beta$-blockers, ACE inhibitors and statins.

Thus, potentially, there exists a risk-treatment mismatch with treatment being withheld from patients who might receive similar or more benefit of longer DAPT.

\section{Comparison of adherence to different DAPT alternatives}

A direct comparison of adherence and treatment length between the different DAPT alternatives after MI is complex because the treatments in clinical practice are prescribed to different patient populations. Even in comparable populations, it is difficult to standardise adherence in a multivariable model as underlying factors (such as tablet pack size and daily dosing patterns) may influence treatment length. Similarly, it is difficult to assess how these underlying factors influence the risk of having a calculated treatment break of 30 days.

Prasugrel is prescribed almost entirely for patients with PCI, whereas clopidogrel and ticagrelor are 
Table 4 Patients with MI discharged in 2012: Cox regression analysis: HR for having a break in $\mathrm{P}_{2} \mathrm{Y}_{12}$ antagonist treatment $>30$ days with or without $\mathrm{PCl}$

\begin{tabular}{|c|c|c|c|c|c|c|}
\hline & \multicolumn{3}{|c|}{ Patients with PCI } & \multicolumn{3}{|c|}{ Non- $\mathrm{PCl}$ patients } \\
\hline & $\begin{array}{l}\text { Number of } \\
\text { patients }\end{array}$ & $\begin{array}{l}\text { Number of } \\
\text { patients with } \\
\text { breaks }\end{array}$ & $\mathrm{HR}(95 \% \mathrm{Cl})$ & $\begin{array}{l}\text { Number of } \\
\text { patients }\end{array}$ & $\begin{array}{l}\text { Number of } \\
\text { patients with } \\
\text { breaks }\end{array}$ & HR $(95 \% \mathrm{Cl})$ \\
\hline All patients & 3373 & 1513 & & 1399 & 972 & \\
\hline Clopidogrel & 697 & 317 & Reference & 579 & 411 & Reference \\
\hline Ticagrelor & 2164 & 937 & 1.20 (1.03 to 1.39$)$ & 800 & 552 & $1.48(1.27$ to 1.72$)$ \\
\hline Prasugrel & 512 & 259 & $1.48(1.24$ to 1.78$)$ & 20 & 9 & $0.86(0.47$ to 1.58$)$ \\
\hline Female & 911 & 398 & Reference & 666 & 481 & Reference \\
\hline Male & 2462 & 1115 & $1.00(0.88$ to 1.13$)$ & 733 & 491 & $0.91(0.78$ to 1.05$)$ \\
\hline$<60$ years & 1216 & 503 & $1.01(0.90$ to 1.14$)$ & 277 & 168 & $1.18(0.97$ to 1.43$)$ \\
\hline 60 to 75 years & 1481 & 648 & Reference & 554 & 344 & Reference \\
\hline$>75$ years & 676 & 362 & $1.15(1.00$ to 1.34$)$ & 568 & 460 & $1.02(0.86$ to 1.21$)$ \\
\hline Local hospital & 878 & 403 & Reference & 498 & 357 & Reference \\
\hline Main regional hospital & 1404 & 620 & $0.97(0.85$ to 1.11$)$ & 588 & 399 & $0.99(0.84$ to 1.18$)$ \\
\hline Tertiary cardiac hospital & 1091 & 490 & $1.00(0.86$ to 1.15$)$ & 313 & 216 & $1.12(0.92$ to 1.38$)$ \\
\hline CABG & 56 & 22 & 0.81 (0.49 to 1.34$)$ & 191 & 107 & $1.00(0.80$ to 1.25$)$ \\
\hline Heart failure & 59 & 42 & $1.40(0.94$ to 2.08$)$ & 86 & 79 & $1.33(0.98$ to 1.81$)$ \\
\hline Stroke & 97 & 56 & 1.34 (0.99 to 1.82$)$ & 101 & 77 & $1.11(0.82$ to 1.49$)$ \\
\hline Atrial fibrillation & 92 & 68 & $1.88(1.41$ to 2.50$)$ & 94 & 81 & $1.27(0.95$ to 1.70$)$ \\
\hline Diabetes & 411 & 229 & $1.23(1.05$ to 1.44$)$ & 258 & 196 & $0.96(0.79$ to 1.16$)$ \\
\hline Cancer & 219 & 131 & $1.09(0.88$ to 1.34$)$ & 125 & 104 & $1.03(0.80$ to 1.33$)$ \\
\hline Major bleeding & 67 & 39 & $1.07(0.74$ to 1.56$)$ & 53 & 36 & $1.20(0.86$ to 1.67$)$ \\
\hline $\begin{array}{l}\text { Peripheral arterial } \\
\text { disease }\end{array}$ & 85 & 61 & $1.63(1.20$ to 2.20$)$ & 93 & 74 & $0.99(0.72$ to 1.36$)$ \\
\hline
\end{tabular}

prescribed irrespective of PCI status. In patients with PCI, the adherence patterns did not differ between the respective DAPTs, whereas non-PCI patients had a generally shorter treatment length and those prescribed ticagrelor showed an increased risk of early treatment break compared with patients prescribed clopidogrel; some of these patients (11\%) were switched to clopidogrel. We did not have access to data that would provide reasons for this shorter treatment length or treatment switch, such as if this switch was carried out in a hospital setting, by general practitioners, or in certain geographical locations. Moreover, a relatively large proportion $(18 \%)$ of patients not undergoing PCI were already on clopidogrel before their MI event, indicating an underlying long-term use not associated with the MI which may explain the longer observed treatment length in this group. In addition, it seems that a larger proportion of clopidogrel patients, both with and without PCI, are treated for more than 12 months after MI.

\section{Strength and limitations}

Our data set is uniquely placed to examine DAPT adherence because it includes nationwide data from all patients hospitalised in Denmark for MI, allowing analyses on a complete and unselected cohort of patients. This reduces potential problems arising from selection bias due to inclusion of selected hospitals, regions or healthcare insurance systems. We believe our results may be generalised to societies with healthcare systems comparable to the one in Denmark. However, this study design also comes with certain limitations. A register-based analysis relies on ICD-10 codes for morbidity data, and therefore the possibility of coding errors cannot be ruled out. Therefore, patients with unstable angina pectoris diagnosis were not included and subcoding into STEMI and NSTEMI was not performed. However, the diagnosis of MI in the Danish National Patient Registry has previously been shown to have both high sensitivity and high specificity. ${ }^{29}$ Further, treatment guidelines for DAPT initiation and treatment duration do not differ between STEMI and NSTEMI. ${ }^{56}$

Since our study is based on central registry data collected primarily for administrative purposes, it is not possible to include clinical data on smoking pattern, weight, blood pressure, laboratory data or socioeconomic status. Furthermore, data on events (recurrent MI, elective PCI, bleedings) during follow-up that might influence treatment length were not included in the current analysis because of the complexity of different patient baseline risks for the DAPTs.

\section{CONCLUSIONS}

The results from this study show that the treatment of patients with MI in Denmark has undergone major changes during 2009-2012. More patients undergo 
invasive procedures (coronary angiography and PCI), and the DAPT pattern has shifted from merely clopidogrel to different treatments for selected patient populations. The majority of patients are discharged with DAPT and the overall treatment length is according to guidelines and in line with what has been observed in randomised controlled clinical trials. Still, there is a proportion of patients not undergoing PCI who are discharged without guideline-recommended DAPT. If treated with DAPT, they have a shorter treatment length. The present findings may indicate the need for more careful attention with regard to DAPT for patients with MI without PCI in Denmark.

\section{Author affiliations \\ ${ }^{1}$ Institute of Applied Economics and Health Research, Copenhagen, Denmark ${ }^{2}$ Department of Clinical Research, OPEN, Odense Patient Data Explorative Network, Odense University Hospital, University of Southern Denmark, Odense, Denmark \\ ${ }^{3}$ Department of Clinical Pharmacology, Institute of Public Health, University of Southern Denmark, Odense, Denmark \\ ${ }^{4}$ AstraZeneca, Södertälje, Sweden \\ ${ }^{5}$ Copenhagen University Hospital Gentofte, Hellerup, Denmark}

Contributors $A G, P H$ and $M E$ were involved in the study design; $A P$ and $A B$ performed the statistical analyses; $A G, P H, T G D, G H G, A P$ and $A B$ were involved in the interpretation of the results; $P H$ and $A G$ wrote the manuscript and $A G, A P, A B, T G D, P H$ and $G H G$ were involved in the critical comments on the manuscript. Mrs Sabrina Imeroski has provided editorial assistance in the preparation of the manuscript.

Funding This work was supported by AstraZeneca (study sponsor). The statistical analysis was agreed on by the study steering committee, and data analysis was performed by the study database owner in collaboration with AstraZeneca. As members of the study steering committee, AstraZeneca took part in the interpretation of data and the drafting of the manuscript. GHG is supported by an unrestricted clinical research scholarship from the Novo Nordisk Foundation.

Competing interests PH and TGD are full-time employees at AstraZeneca. ME and $A G$ are employed by the Institute of Applied Economics and Health Research. GHG reports research grants from AstraZeneca, Pfizer, Bristol-Myers Squibb and Bayer. AP reports funding from Servier, Boehringer-Ingelheim, Astellas, AstraZeneca, Almirall and Alcon.

Provenance and peer review Not commissioned; externally peer reviewed.

Data sharing statement No additional data are available.

Open Access This is an Open Access article distributed in accordance with the Creative Commons Attribution Non Commercial (CC BY-NC 4.0) license, which permits others to distribute, remix, adapt, build upon this work noncommercially, and license their derivative works on different terms, provided the original work is properly cited and the use is non-commercial. See: http:// creativecommons.org/licenses/by-nc/4.0/

\section{REFERENCES}

1. Dudas K, Lappas G, Rosengren A. Long-term prognosis after hospital admission for acute myocardial infarction from 1987 to 2006. Int J Cardiol 2012;155:400-5

2. Fox KAA, Dabbous $\mathrm{OH}$, Goldberg RJ, et al. Prediction of risk of death and myocardial infarction in the six months after presentation with acute coronary syndrome: prospective multinational observational study (GRACE). BMJ 2006;333:1091-4.

3. Gulliksson M, Wedel H, Köster M, et al. Hazard function and secular trends in the risk of recurrent acute myocardial infarction: 30 years of follow-up of more than 775000 incidents. Circ Cardiovasc Qual Outcomes 2009;2:178-85.

4. Jernberg $\mathrm{T}$, Johanson $\mathrm{P}$, Held $\mathrm{C}$, et al. Association between adoption of evidence-based treatment and survival for patients with ST-elevation myocardial infarction. JAMA 2011;305:1677-84

5. Hamm CW, Bassand JP, Agewall S, et al. ESC Guidelines for the management of acute coronary syndromes in patients presenting without persistent ST-segment elevation: the task force for the management of acute coronary syndromes (ACS) in patients presenting without persistent ST-segment elevation of the European Society of Cardiology (ESC). Eur Heart J 2011;32:2999-3054.

6. Steg PG, James SK, Atar D, et al. ESC Guidelines for the management of acute myocardial infarction in patients presenting with ST-segment elevation. Task force on the management of ST-segment elevation acute myocardial infarction of the European Society of Cardiology (ESC). Eur Heart J 2012;33:2569-619.

7. Sørensen R, Gislason GH, Fosbøl EL, et al. Initiation and persistence with clopidogrel treatment after acute myocardial infarction: a nationwide study. Br J Clin Pharmacol 2008;66:875-84.

8. EMA (2015-08-11) Ticagrelor (Brilique) Summary of product characteristics. European Medicines Agency. http://www.ema.europa eu/docs/en GB/document library/EPAR - Product Information/ human/001241/WC500100494.pdf (accessed 1 Sep 2015).

9. EMEA (2014-02-17) Prasugrel (Efient) Summary of product characteristics. http://www.ema.europa.eu/docs/en GB/document library/EPAR_-_Product_Information/human/000984/WC500021971. pdf (accessed 1 Jun 2015).

10. The national Danish ACS guidelines. http://nbv.cardio.dk/ (accessed 1 Jun 2015)

11. Thygesen LC, Ersbøll AK. Danish population-based registers for public health and health-related welfare research: introduction to the supplement. Scand J Public Health 2011;39:8-10.

12. Kildemoes HW, Sørensen HT, Hallas J. The Danish Nationa Prescription Registry. Scand J Public Health 2011;39:38-41.

13. Anatomic Therapeutic Chemical (ATC) index. http://www.whocc.no/ filearchive/publications/1 2013guidelines.pdf (accessed 1 Jun 2015)

14. Lynge E, Sandegaard JL, Rebolj M. The Danish National Patient Register. Scand J Public Health 2011;39:30-3.

15. Pedersen CB. The Danish Civil Registration System. Scand J Public Health 2011;39:22-5

16. Thygesen LC, Daasnes C, Thaulow I, et al. Introduction to Danish (nationwide) registers on health and social issues: structure, access, legislation, and archiving. Scand J Public Health 2011;39:12-16.

17. Nordic Medico-Statistical Committee: Classification of Surgical Procedures, version 1.15. Copenhagen, Denmark 2010: Publication 93

18. Gislason GH, Rasmussen JN, Abildstrøm SZ, et al. Long-term compliance with beta-blockers, angiotensin-converting enzyme inhibitors, and statins after acute myocardial infarction. Eur Heart $J$ 2006;27:1153-8.

19. Mårtensson S, Gyrd-Hansen D, Prescott E, et al. Trends in time to invasive examination and treatment from 2001 to 2009 in patients admitted first time with non-ST elevation myocardial infarction or unstable angina in Denmark. BMJ Open 2014;4:e004052.

20. Fokkema ML, James SK, Albertsson P, et al. Population trends in percutaneous coronary intervention: 20 -year results from the SCAAR (Swedish Coronary Angiography and Angioplasty Registry). J Am Coll Cardiol 2013;61:1222-30.

21. Vamos EP, Millett C, Parsons C, et al. Nationwide study on trends in hospital admissions for major cardiovascular events and procedures among people with and without diabetes in England, 2004-2009. Diabetes Care 2012;35:265-72.

22. SWEDEHEART 2014 Annual Report (English edition). http://www.ucr. uu.se/swedeheart/index.php/arsrapporter (accessed 18 May 2015).

23. Erne P, Gutzwiller F, Urban P, et al. Characteristics and outcome in acute coronary syndrome patients with and without established modifiable cardiovascular risk factors: insights from the nationwide AMIS Plus Registry 1997-2010. Am J Cardiol 2013;111:202-7.

24. Bajaj RR, Goodman SG, Yan RT, et al. Treatment and outcomes of patients with suspected acute coronary syndromes in relation to initial diagnostic impressions (insights from the Canadian Global Registry of Acute Coronary Events [GRACE] and Canadian Registry of Acute Coronary Events [CANRACE]). Am J Cardiol 2013;111:202-7.

25. Jortveit J, Govatsmark RE, Digre TA, et al. Myocardial infarction in Norway in 2013. Tidsskr Nor Laegeforen 2014;134:1841-6.

26. Jernberg $\mathrm{T}$, Hasvold $\mathrm{P}$, Henriksson $\mathrm{M}$, et al. Cardiovascular risk in post-myocardial infarction patients: nationwide real world data demonstrate the importance of a long-term perspective. Eur Heart $J$ 2015;36:1163-70.

27. Wiviott SD, Braunwald E, McCabe $\mathrm{CH}$, et al. Prasugrel versus clopidogrel in patients with acute coronary syndromes. $N$ Engl J Med 2007;357:2001-15.

28. Wallentin L, Becker RC, Budaj A, et al. Ticagrelor versus clopidogrel in patients with acute coronary syndromes. $N \mathrm{Engl} J \mathrm{Med}$ 2009;361:1045-57.

29. Schmidt M, Schmidt SAJ, Sandegaard JL, et al. The Danish National Patient Registry: a review of content, data quality, and research potential. Clin Epidemiol 2015;7:449-90. 